\title{
Do the Chloroplasts Exhibit Sexuality?
}

\author{
Lucian Gavrilă, Gr. Mihăescu and FI. Tăcină \\ Genetics Department, Faculty of Biology, Bucharest University, \\ Aleea Portocalilor 1-3, sector 6, Bucharest, 77-206, România
}

Accepted October 15, 1986

The chloroplasts represent the most sophisticated type of plastids (Walker 1970) and, according to the modern view regarding the orgins of eukaryotic cell (Margulis 1971, Stanier 1974, Taylor 1974, Woesse 1977, Gray and Doolittle 1982), the modern mitochondria and chloroplasts are derived from free-living prokaryotic microorganisms through serial endosymbiosis, some two billions years ago.

Early, in 1905, Mereschowsky (citted by Margulis 1971) was the first to consider the exogenic origin of chloroplasts. Many investigators considered free-living cyanobacteria as potential ancestors for modern chloroplasts but, since the discovery of photosynthetic bacteria like those belonging to the genus Prochloron, it is considered that, at least for chlorophycean algae and high-plants chloroplasts, the ancestors might be such non-cyanobacterial photosynthetic microorganisms (Lewin 1981).

It has been assumed that one of the first steps in evolution of endosymbionts in the hostcell was the loss of their ability to synthesize peptidoglicans, the main component of the cellwall. Finally, a structural modification in the genetic system of endosymbionts led to their dependence on host-cell, with the transfer of some genetic determinants of endosymbionts to nuclear host-genome. In such a way, the endosymbionts have lost their organismus status, acquiring instead the organelle status. It is not a surprise to find in modern chloroplasts some features of their ancestors living as free organisms.

In the last two decades, intensive investigations have been carried out in Euglena which has proved to be a very interesting objective for biological investigations at molecular and cellular levels (Leedale 1971, Buetow 1968). Our own investigations in Euglena terricola allowed us to evidentiate the phenomenon of chloroplast fusion, which we consider to represent a reminiscence of sexual potentiality exhibited by chloroplast ancestors while they have been free-living organisms. This aspect might be very important in substatiating the arguments supporting the endosymbiotic origin of modern chloroplasts.

\section{Materials and methods}

Discovered just accidentally in the period 1975-1976 in one cell of E. terricola, the imagine of chloroplast fusion was published in our book of algal genetics (Gavrilă 1978), without any interpretation of the phenomemon. Later, we have repeatedly found such aspects in this photosynthetic microorganism and our attention has been focussed on it.

\section{Results}

In E. terricola, as well as in other Euglenoids, the chloroplasts are of the lamella type, without grana. They are surrounded by a sheat (Fig. 1) which is called peristomium. The number of lamellae is varible, of the figure 10 to 15 per chloroplast and a lamella is usually composed of 3, rarely 2-5 tightly associated thylakoids. The stroma, fundamental matrix of the chloroplast, exhibits a granular aspect due to the presence of a rich population of plastidial ribosomes. 


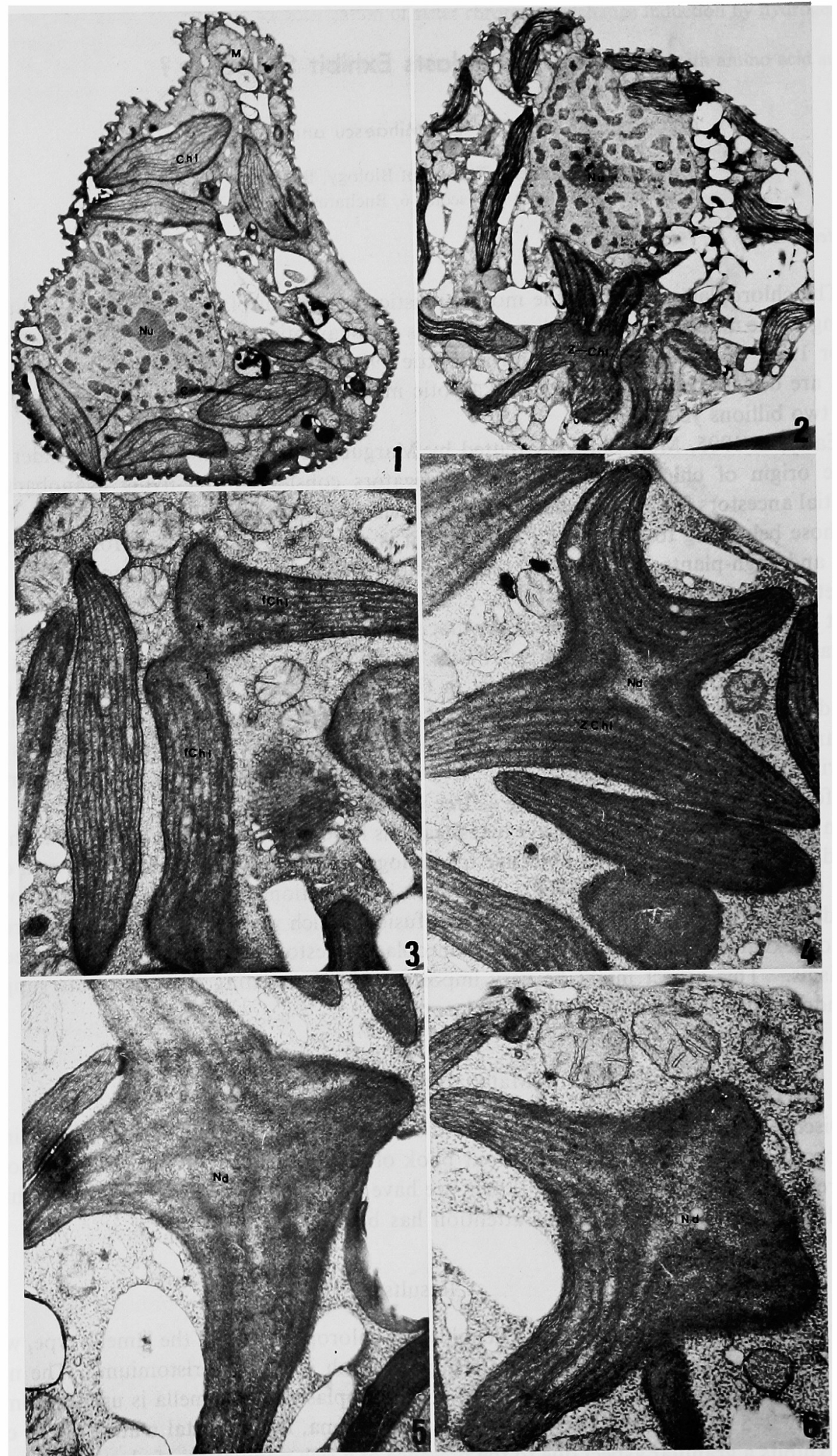


The plastidial genophore is represented by randomly dispersed chloroplastic DNA fibrils located in the chloroplast matrix.

The fusion of chloroplasts in $E$. terricola has been repeatedly encountered in natural populations as well as in laboratory cultures (Fig. 2). In order to eliminate the eventuality that the presence of some endoparasitical microorganisms, described in this species by Gavrilă (1978) and Gavrila et al. (1981) would be responsible for the chloroplast fusion, we have performed a thoroughly cytological analysis of the Euglena populations which have been worked out and exhibited chloroplast fusion phenomenon. We have also eliminated the possibility that the sensecence would be responsible for such fusion phenomenon, analysing young, old and senescent cultures, finding a high incidence of chloroplast fusion in young cultures, higher than in old or senescent ones. The highest incidence of chloroplast fusion has been encountered in natural populations. In this way, we have reached the conclusion that the chloroplast fusion phenomenon encountered in E. terricola is a normal, physiological aspect of its cell biology. Extensive electron microscopical investigations in $E$. terricola focussed on this phenomenon allowed us to follow it in almost all its steps. It was not a surprise for us to find similar aspects regarding the chloroplast fusion in some flowering plants, in high metabolically active cells, but not in such extension as in this unicellular photosynthetic alga $E$. terricola.

The chloroplast fusion in E. terricola is a high-organized process under the control of genetic apparatus of the chloroplast itself, implying a rigourous sequence of events. The chloroplasts which are to fuse enter firstly what we can call "the competence state" in which local or general modifications (i.e. disappearance) of peristomium takes place. Such chloroplasts are coming in contact (Fig. 3), establishing later a true synapsis (Fig. 4), their lamella system being profoundly modified, loosing their structural individuality, while the stroma becomes fluidic. Usually, in the fusion process are participating only two chloroplasts, respecting the universal principle of the sexuality process. Such fusing chloroplasts can be regarded as being of opposite "sex". Figure 2 reveals two fused chloroplasts having " $H$ " aspect, but near them, in close vicinity, one side and the other, there are also two chloroplasts looking like they are ready to participate in the sexual process (i.e. in the fusion process), exhibiting a "competence state" i.e. with obvious apical fluidity of their peristomium and lamella system which makes them ready to fuse when every one will find its partner of opposite "sex". It is very clear from this picture that such "competent" chloroplasts are oriented toward the fusing chloroplasts, but they do not participate in the fusion process. They will fuse when they will come in contact. In Euglena, such "competent" chloroplasts will ready come in contact because of its specific movements that facilitate the movement of the all organelles inside the cell and, in this way, their permanent contact and separation. Such specific movements of Euglena cell can be performed because this species, as well as some other Euglenoids, lacks a rigid cellwall, having instead a flexible surrounding pellicular layer of a crenelated appearance.

Figs. 1-6. 1, the fine structure of an Euglena terricola cell which does not exhibit chloroplast fusion. $\mathrm{M}$ : mitochondrion, $\mathrm{Chl}$ : chloroplast, $\mathrm{Nu}$ : nucleolus, $\mathrm{C}$ : chromosomes. $\times 9,000.2$, the fine structure of an E. terricola cell from the same population as the cell in Fig. 1. This cell exhibits chloroplast fusion phenomenon. As a consequence of chloroplast fusion a zygote-chloroplast (Z-Chl) has arised. Note the presence of other two chloroplasts being in a presumed "competence" state, one on the left side, the other on the right side of the zygote-chloroplast. $\times 9,500$. 3 , an end-to-end fusion of two "competent" chloroplasts ( $\mathrm{f}-\mathrm{Chl}) . \times 30,000,4$, a zygote-chloroplast (Z-Chl) resulted through a side-by-side fusion of two "competent" chloroplasts by their convex parts. Note the bacterial appearance of the condensed chloroplast nucleoid (Nd) of the zygotechloroplast. $\times 36,000.5$ and 6 , zygote-chloroplast, long time after fusion. The lamellar system has almost lost its regular appearance. The chloroplast DNA fibrils of these two chloroplasts are mixed up, resulting a nucleoidal structure $(\mathrm{Nd})$, having a granular-fibrilar appearance. $\times 40,000$ and 36,000 respectively. 
The chloroplasts are fusing in an end-to-end (Fig. 3) or side-by-side (Fig. 4) manner. It is of great significance that the chloroplast fusion is followed by the concentration of their randomly dispersed into the stroma DNA fibrils, taking an appearance which resembles a

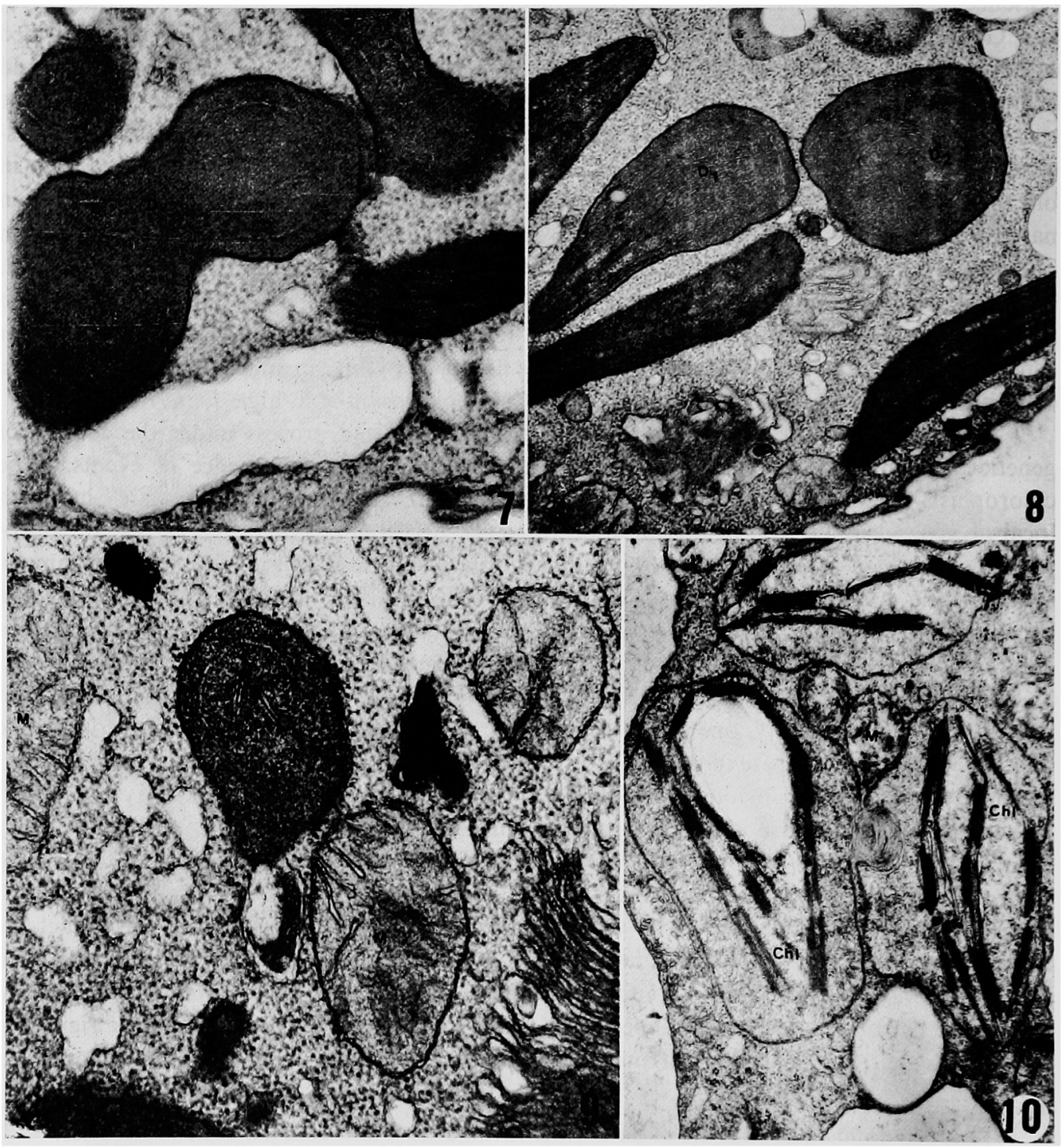

Figs. 7-10. 7, a dividing zygote-chloroplast (Dv) by a median constriction mechanism. $\times 18,000$. 8 , two daughter-proplastids $\left(D_{1}\right.$ and $\left.D_{2}\right)$ resulted by division of a zygote-chloroplast. $\times 20,000$. 9, a proplastid $\left(P_{p}\right)$ resulted after the division of the zygote-chloroplast. Note the reorganization of lamellar system, This proplastid can not be confused with mitochondria $(M) . \times 50,000.10$, the interaction between two chloroplasts in a nectariferous gland cell of Robinia pseudacacia, resembling a sexual process. $\times 40,000$.

nucleoidal structure (Figs. 4-6) which is commonly encountered in bacterial or cyanobacterial cells. It means that the chloroplast fusion facilitates their genetic recombination, and this might be the sense of the fusion process. Such recombinational event is perhaps required for the recovery of the organelles after an intense use of their synthetic potential. 
The fused chloroplasts exhibit a fine structure completely different from that encountered in unfused ones from the same cell (Fig. 3). After fusion, the hybrid or zygote chloroplast undergoes a uniformization (i.e. fluidization) of its structure. This zygote chloroplast exhibits afterwards the appearance of a typical prokaryotic cell which divides by median constriction (Fig. 7). The zygote chloroplast corresponds to a dedifferentiation state, which probably was characteristic for the ancestors of modern chloroplasts, when they have been free-living organisms. The homogenization of zygote chloroplast structure might be required by the recombination process itself. The dedifferentiation step in evolution of zygote chloroplast is followed by a reorganization of lamella system. By median constriction of zygote chloroplast, two daughter-chloroplasts are resulting (Fig. 8), which at beginning exhibit a characteristic proplastid structure, well defined in some instances (Fig. 9).

In Robinia pseudacacia, among higher plants, in the ontogenetic development of nectariferous glands, we have also encountered in some cells a local invagination-evagination of two chloroplasts that happened to be in a very close vicinity. In the contact area, multilamellar bodies in the invagination cavity are organized (Fig. 10). In the flowering plants, as well as in the monocellular photosynthetic microorganisms, the contact between chloroplasts of the opposite "sex" is much more difficult to be established due to the rigidity of the pectocellulosic cell wall. The spectacular aspects of the chloroplast fusion in Euglena are based on their easiness to come in contact as a consequence of the flexibility of pellicular layer and rapid movements of metaboly which are peculiar features of this organism.

Taking in account that the fusion of the chloroplasts is performed by healthy cells, being more frequent in young cultures that are actively photosynthetising in Euglena, and on the other side it has been encountered in high metabolically active cells from nectariferous glands in Robinia pseudacacia, we interpret such a phenomenon as a sexual-like process involved in the genetic recombination of the chloroplasts and representing a reminescence of sexuality which has been performed by the ancestors of modern chloroplasts while they have been free-living organisms. The role of such "sexual" process in chloroplasts might be related with the necessity for recovering of the organelles which have been engaged in a very high synthetic activity. Through such a "sexual" process, the chloroplasts perform genetic recombination, recovering their synthetic potential.

This descovery of sexual process in chloroplasts might have profound implications in the research on cell biology as well as in our knowledge regarding the photosynthesis phenomenon and other chloroplast functions.

\section{References}

Buetow, D. E. 1968, Euglena-cells for biological investigation. In The Biology of Euglena (D.E. Buetow ed.). Acad. Press, New York, London.

Gavrilă, L. 1978. Algal Genetics. Publishing House of Romanian Academy of Sciences, Bucharest: 1-141 -, Soran, V., Bercea, V., Ahmad, S. and Spârchez, C. 1981. The genetic organization in Dinoflagellates and Euglenoids. A comparative cytogenetic and cytophotometric study. Cytologia 46: 1-13.

Gray, M. W. and Doolittle, W. F. 1982. Has the endosymbiont hypothesis been proven? Microbiol, Rev. 46: $1-42$.

Leedale, G. F. 1971, The Euglenoids. Oxford University Press

Lewin, R. A. 1981, Prochloron and the theory of symbiogenesis. Ann. N. Y. Academy of Sciences. No. 161: $1020-1022$,

Margulis, L. 1971. The origin of plant and animal cells. Amer. Sci. 59: 230-235.

Stanier, R. Y. 1974. The origins of photosynthesis in eukaryotes, Symposium of the 24th Society for General Microbiology. Evolution in the Microbial World. Cambridge Univ. Press, London, 219-258.

Taylor, I. J. R. 1974. Implications and extensions of the serial endosymbiosis theory for the origin of eukaryotes. Taxon 23: 229-258.

Walker, D. 1970 . Three phases of chloroplast research. Nature (London). 226: 1204-1208.

Woesse, C. R. 1977. Endosymbionts and mitochondrial origins, J. Molec. Evol. 10: $93-96$. 